\title{
Genome-wide analysis of Jatropha curcas MADS-box gene family and functional characterization of the JCMADS40 gene in transgenic rice
}

Yuehui Tang ${ }^{1,2^{*}+}$ D, Jian Wang ${ }^{1,2+}$, Xinxin Bao ${ }^{3}$, Qian Wu' ${ }^{1}$, Tongwen Yang ${ }^{1}$, Han $\mathrm{Li}^{1}$, Wenxia Wang ${ }^{1}$, Yizhen Zhang ${ }^{1}$, Nannan Bai ${ }^{1}$, Yaxin Guan ${ }^{1}$, Jiaxi Dai ${ }^{1}$, Yanjie Xie ${ }^{1}$, Shen $\mathrm{Li}^{1}$, Rui Huo ${ }^{1}$ and Wei Cheng ${ }^{1}$

\begin{abstract}
Background: Physic nut (Jatropha curcas), an inedible oilseed plant, is among the most promising alternative energy sources because of its high oil content, rapid growth and extensive adaptability. Proteins encoded by MADS-box family genes are important transcription factors participated in regulating plant growth, seed development and responses to abiotic stress. However, there has been no in-depth research on the MADS-box genes and their roles in physic nut.

Results: In our study, 63 MADS-box genes (JCMADSs) were identified in the physic nut genome, and classed into five groups (MIKC, Ma, M $\beta, M Y$, MIKC*) according to phylogenetic comparison with Arabidopsis homologs. Expression profile analysis based on RNA-seq suggested that many JCMADS genes had the strongest expression in seeds, and seven of them responded in leaves to at least one abiotic stressor (drought and/or salinity) at one or more time points. Transient expression analysis and a transactivation assay indicated that JCMADS40 is a nucleuslocalized transcriptional activator. Plants overexpressing JCMADS40 did not show altered plant growth, but the overexpressing plants did exhibit reductions in grain size, grain length, grain width, 1000-seed weight and yield per plant. Further data on the reduced grain size in JCMADS40-overexpressing plants supported the putative role of JCMADS genes in seed development.

Conclusions: This study will be useful in order to further understand the process of MADS-box genes involved in regulating growth and development in addition to their functions in abiotic stress resistance, and will eventually provide a theoretical basis for the functional investigation and the exploitation of candidate genes for the molecular improvement of physic nut.
\end{abstract}

Keywords: Physic nut, MADS-domain proteins, JCMADS40, Abiotic stress, Seed size, Transgenic plants

\footnotetext{
* Correspondence: yhtang2005@163.com

†Yuehui Tang and Jian Wang contributed equally to this work.

${ }^{1}$ Key Laboratory of Plant Genetics and Molecular Breeding, Zhoukou Normal University, Henan, Zhoukou, China

${ }^{2}$ Henan Key Laboratory of Crop Molecular Breeding and Bioreactor, Henan, Zhoukou, China

Full list of author information is available at the end of the article
}

(c) The Author(s). 2020 Open Access This article is licensed under a Creative Commons Attribution 4.0 International License, which permits use, sharing, adaptation, distribution and reproduction in any medium or format, as long as you give appropriate credit to the original author(s) and the source, provide a link to the Creative Commons licence, and indicate if changes were made. The images or other third party material in this article are included in the article's Creative Commons licence, unless indicated otherwise in a credit line to the material. If material is not included in the article's Creative Commons licence and your intended use is not permitted by statutory regulation or exceeds the permitted use, you will need to obtain permission directly from the copyright holder. To view a copy of this licence, visit http://creativecommons.org/licenses/by/4.0/. The Creative Commons Public Domain Dedication waiver (http://creativecommons.org/publicdomain/zero/1.0/) applies to the data made available in this article, unless otherwise stated in a credit line to the data. 


\section{Background}

The regulation of plant growth, development and stress responses is complex and is coordinated by many mechanisms. These mechanisms are under the control of many related genes acting through complex regulatory networks. In these processes, transcription factor (such as members of the MYB, HD-Zip, ARF, NAC, MADSbox, and ERF gene families) specifically recognize cisregulatory elements present in the promoter regions of these genes, and regulate their expression so as to modulate a wide range of biochemical, physiological and developmental processes [1-6].

The MADS-domain proteins constitute one of the largest families of transcription factors. Structurally, almost all MADS-domain proteins contain a highly conserved DNA binding domain containing 58 amino acids residues, which can bind to DNA at consensus recognition sequences known as CC(A/T)6GG (CArG boxes), regulating the transcription of downstream genes [7]. Other domains are also present in the MADS-domain proteins; they include the keratin-like (K) domain, which is responsible for dimerization, the I (intervening) domain, which is capable of binding to form dimers, and the $\mathrm{C}$ (C-terminal) region, which is highly variable [8]. According to the similarities of amino acid sequences and structural features of the conserved domains, MADSdomain proteins in Arabidopsis can be classified into five groups (designated MIKC $C^{C}, M \alpha, M \beta, M \gamma, M I K C^{*}$ ) [9].

In recent years, many MADS-domain proteins have been widely identified and characterized, by means of genome-wide and expression profiles analysis researches, from a number of plant species including rice [10], wheat [11], potato [12], moso bamboo [13], Arabidopsis [9] and sheepgrass [14]. Studies on overexpression and mutant plants in dicots and monocots have suggested that members of MADS-box family play crucial roles in controlling plant growth and development [1]. For example, the abs stk double mutant has a reduced number of fertilized ovules and undergoes seed abortion resulting in very few seeds [15]. In rice, OsMADS87 RNAi lines produce smaller seeds and overexpression (OE) lines have larger seeds compared with wild-type seeds [16]. The Arabidopsis agl62 mutant shows accelerated endosperm cellularization, showing that AGL62 plays an important regulatory role in inhibiting cellularization [17]. The svp and flm mutants exhibit temperatureinsensitive flowering across wide temperature ranges [18], while FUL is necessary for leaf and fruit development and flowering in Arabidopsis [19]. SEP1, SEP2, and SEP3 have been proven to be critical for the development of carpels, stamens, and petals [20]. In addition to participating in regulating plant growth, and development, evidence is accumulating to suggest that MADSbox genes also participated in plant responses to salt and drought stresses. For example, CaMADS-overexpressing Arabidopsis plants show increased resistance of salinity and cold stresses [21]. Loss-of-function mutations in $S V P$ result in sensitivity to drought stress, whereas the $S V P$-overexpressing plants are more tolerant [22]. In tomato, SIMBP11-RNAi plants are less tolerance in response to salinity stress, but overexpressing this MADS-box gene confers salt stress tolerance [23]. However, although the functions of various members of MADS-box family have been made clear, little is known about the genes of this family and their roles in numerous taxa, containing the Euphorbiaceae.

Physic nut, a small woody member of the Euphorbiaceae, is an inedible oilseed plant grown mainly in tropical and subtropical regions and the oil in its seeds is used widely in industry [24]. Importantly, it has drawn much attention because it is one of the most suitable plants for producing biodiesel owing to its fast growth, ease reproduction, high oil content and wide adaptability [24]. Further research is thus necessary in order to clarify the molecular mechanisms of key genes in regulating physic nut development. The recent release of the physic nut genome sequence allows us to explore all the $J_{c M A D S}$ genes at the genome level. However, there has as yet been no systematic study on the identities, expression patterns and functions of physic nut's MADS-box genes in physic nut. To fill the deficiency, we firstly searched for and confirmed 63 MADS-box genes in physic nut (thereafter referred to as JcMADS genes). Secondly, we investigated their phylogenetic relationships, conserved motifs, chromosomal distribution, expression profiles and potential roles in physic nut development. Finally, JcMADS40 was chosen for further functional analysis, and we tested its effects in transgenic rice. This study focuses on the functional roles of JcMADS genes in the development of physic nut.

\section{Results}

\section{Identification of MADS-domain proteins in physic nut}

All Arabidopsis MADS-domain protein sequences were used as queries in a BlastP search to identify physic nut proteins. A Hidden Markov Model (HMM) search was also performed against the protein database from physic nut by using the MADS-domain PF00319. In total, 63 putative MADS-domain proteins (designated JcMADS01 to JcMADS63) were identified in physic nut, with the presence of the MADS-domain in each of them being confirmed by a SMART website search. The open reading frame (ORF) lengths of 63 JcMADS genes ranged from $195 \mathrm{bp}$ (JcMADS35) to $1164 \mathrm{bp}$ (JcMADS34), thus potentially the proteins encoded would be from 64 to 387 amino acids; their GenBank accession numbers are given in Additional File 1. 


\section{Phylogenetic relationships of the JcMADS proteins}

To clarify the phylogenetic relationships of the physic nut MADS family proteins with the previously reported members of the family in Arabidopsis and rice, an unrooted tree was constructed by IQ-TREE using the Maximum likelihood method (Fig. 1). On the basis of the similarity of full-length amino acid sequences, we subdivided the 246 typical members of the MADS gene family into 5 groups (designated $M I K C^{C}, M \alpha, M \beta, M \gamma$, MIKC*), according to the previous classification of MADS proteins from Arabidopsis [9, 25]. It was worth mentioning that the $M \alpha, M \beta$ and $M \gamma$ belonged to type I, whereas the type II group contained MIKC** and MIKC ${ }^{\mathrm{C}}$. Furthermore, the MIKC ${ }^{\mathrm{C}}$ proteins, which could be further divided into 12 subfamilies (Fig. 1), based on the previous classification of MADS proteins [25]. Of the 63 inferred physic nut JcMADS proteins (Additional File 2), thirty-two were assigned to group MIKC ${ }^{\mathrm{C}}$ (JcMADS32JcMADS63), thirteen to group M $\alpha$ (JcMADS19JcMADS31), four to group M $\beta$ (JcMADS01-JcMADS04), six to group $\mathrm{M \gamma}$ (JcMADS05-JcMADS10) and eight to group MIKC* (JcMADS11-JcMADS18). In the phylogenetic tree, some members of the JcMADS gene family formed related sister pairs (Fig. 1): JcMADSO2 and 03, 06 and 07,14 and 15, 19 and 20,22 and 23, 38 and 39 . There were also triplets (JcMADS08, 09 and 10; 28, 30 and $31 ; 40,41$ and 42 ). The tree indicated that proteins in group $\mathrm{MIKC}^{\mathrm{C}}$ were the most numerous; it contained 39 AtMADS, 38 OsMADS and 32 JcMADS proteins.

\section{Conserved motifs in JcMADS proteins}

The structures of proteins encoded by JcMADS genes were analyzed using the MEME online software tool. Twenty conserved motifs, which we named motifs $1-20$, were identified in the 63 JcMADS proteins (Fig. 2 and Additional File 3). As expected, motif 1 and motif 4 corresponded to the typical MADS-domain, and motif 1 was found in all the JcMADS proteins. Motif 9, specifying the $\mathrm{K}$ domain, was found in most $\mathrm{MIKC}^{\mathrm{C}}$ type proteins; the exceptions were JcMADS35, 43, 47, 50, 54 and 62 , which have relatively short amino acid sequences. In addition to motifs with known functions, some motifs

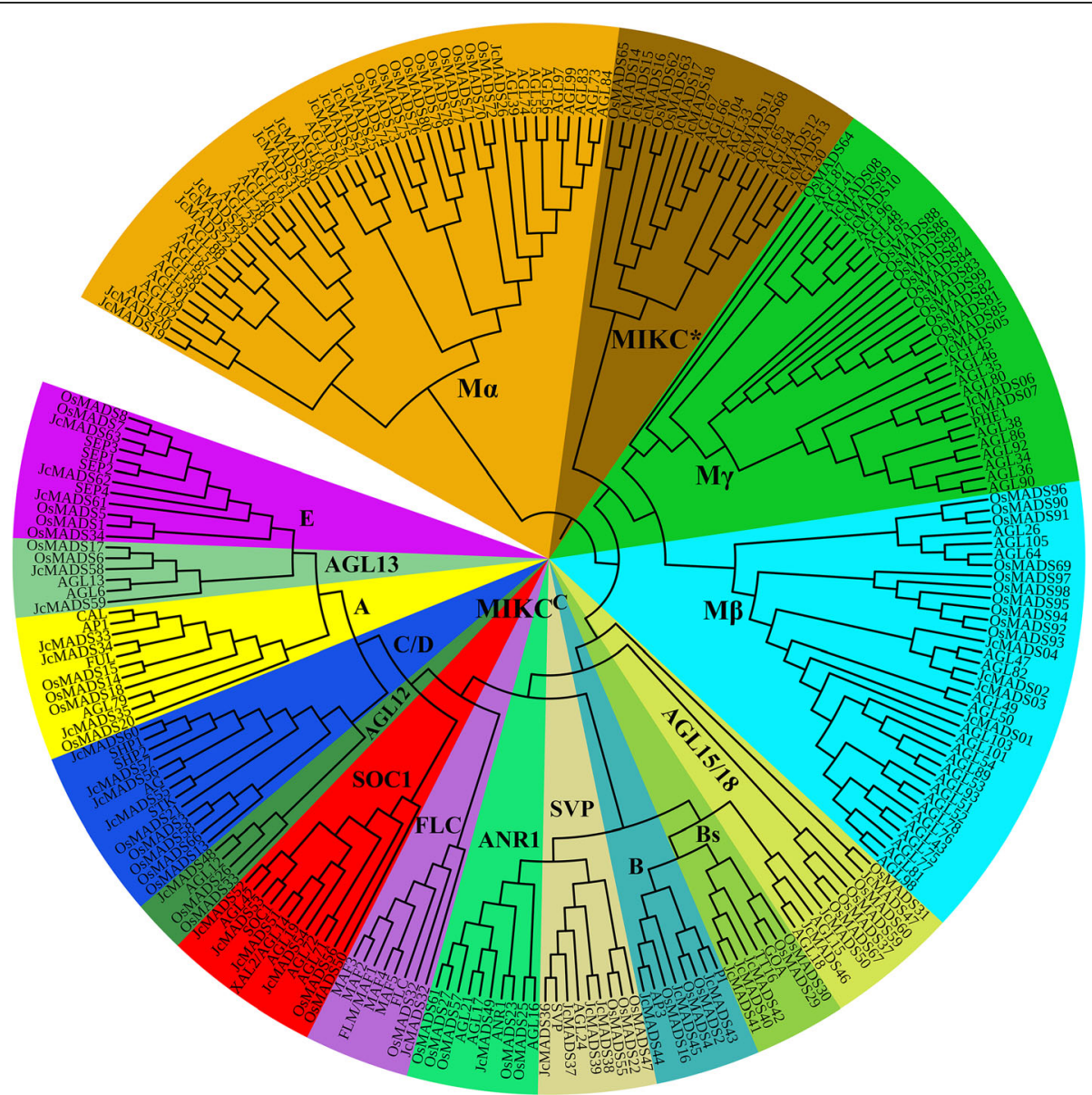

Fig. 1 Unrooted phylogenetic tree of the MADS-box family proteins in physic nut, rice and Arabidopsis. The phylogenetic tree was constructed by IQ-TREE using the Maximum likelihood method 


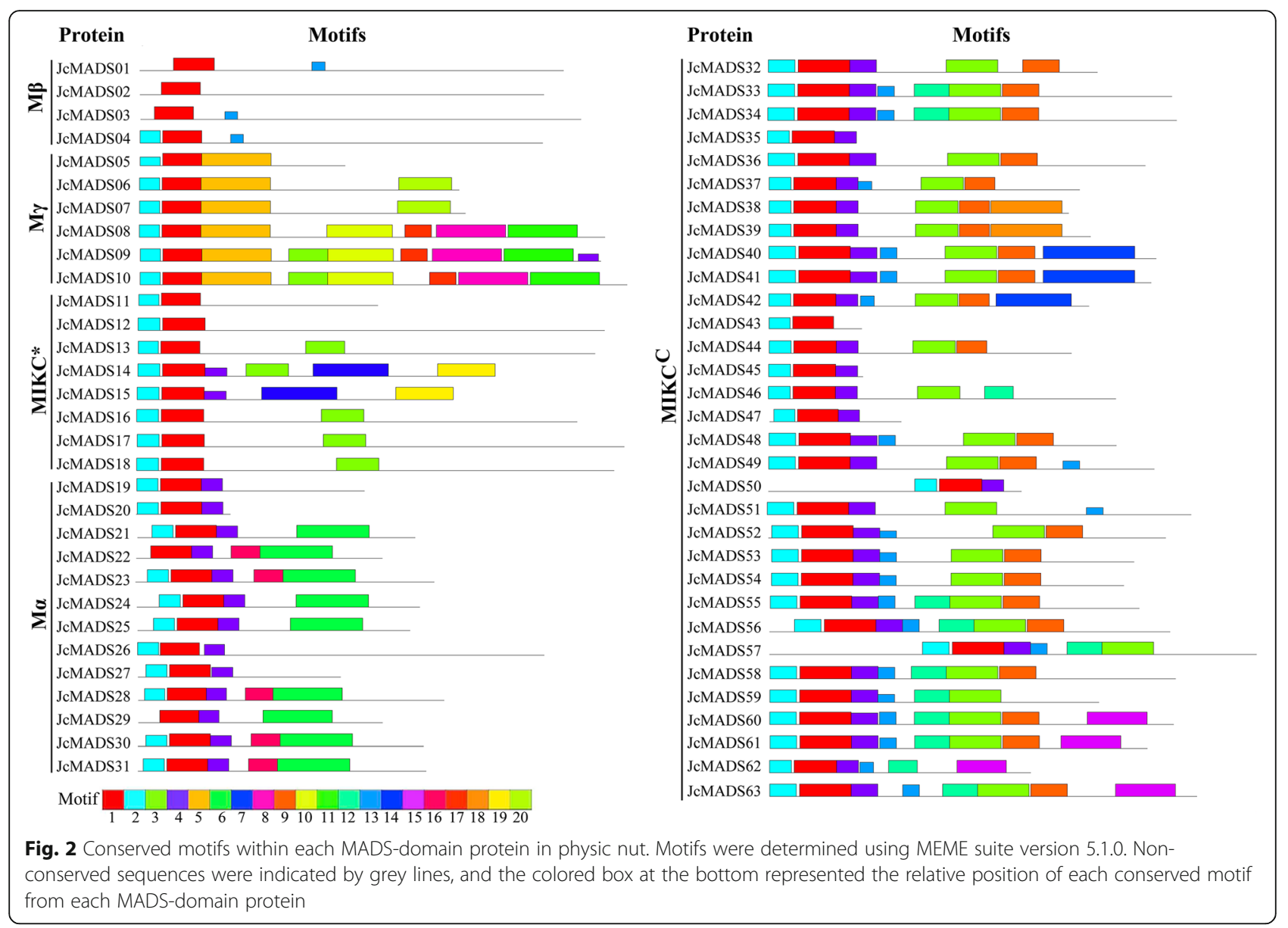

with unknown functions have also been detected. For example, motifs 5, 10 and 20 were observed only in group $\mathrm{M} \gamma$, whereas motif 13 was found only in groups $\mathrm{MIKC}^{\mathrm{C}}$ and $\mathrm{M} \beta$. Motif 12 was found only in group MIKC $^{\mathrm{C}}$, while motifs 14 and 19 was detected only in group MIKC* Additionally, most conserved motifs detected in JcMADS proteins were clade-specifically assigned in different groups, suggesting similarity of function within a given group.

\section{Chromosomal localization of JCMADS genes}

A total of $62 J c M A D S$ genes, except JcMADS45, were mapped to linkage groups (LGs) based on a previously published report of physic nut [26]. As shown in Fig. 3, we found that LGs 4 and 7 had more members of the JcMADS gene family than other LGs, with eleven and nine $J C M A D S$ genes respectively. They were followed by LGs 2, 3, 5 and 10, each of which had six JcMADS genes. In addition, there was five $J_{c M A D S}$ genes on each of LGs 6 and 9, three on LG8, three on LG11 and two on LG1. The results also indicated that most JcMADS genes were located in the lower and middle part from the LGs. Tandem duplications, defined as tandem repeats which are separated by $<4$ non-homologous spacers or are genes located within $50 \mathrm{~kb}$ of each other [27], were found among these members of the JcMADS gene family. The gene pairs present as tandem repeats $(\mathrm{T})$ were T1 (JcMADS41 and 42), T2 (JcMADS28 and 39), T3 (JcMADS22, 23 and 56), T4 (JcMADS33 and 61), T5 (JcMADS52 and 59), T6 (JCMADS12 and 62), T7 (JcMADS10 and 27), T8 (JcMADS47 and 63), T9 (JcMADS29 and 49), T10 (JcMADS53 and 58), T11 (JcMADS02, 35 and 60) and T12 (JcMADS30, 31 and 50), on LG2, LG2, LG3, LG4, LG5, LG5, LG6, LG7, LG7, LG8, LG9 and LG10 respectively.

\section{Expression profile of JCMADS genes under non-stressed growth condition}

To clarify the roles of the JcMADS in regulating physic nut development, we examined the expression profiles of $J_{c M A D S}$ genes in roots, stem cortex, leaves, and seeds (S1 and S2) under non-stressed growth conditions based on data from RNA sequencing (RNA-seq) (Additional File 4 and Fig. 4). The result suggested that fifty of the predicted JCMADS genes were expressed in at least one of the organs examined, while thirteen (JcMADSO4, 11, 17, 20, 21, 22, 28, 30,31, 35, 43, 45 and $57)$ were not expressed in any of these tissues. Of the 50 


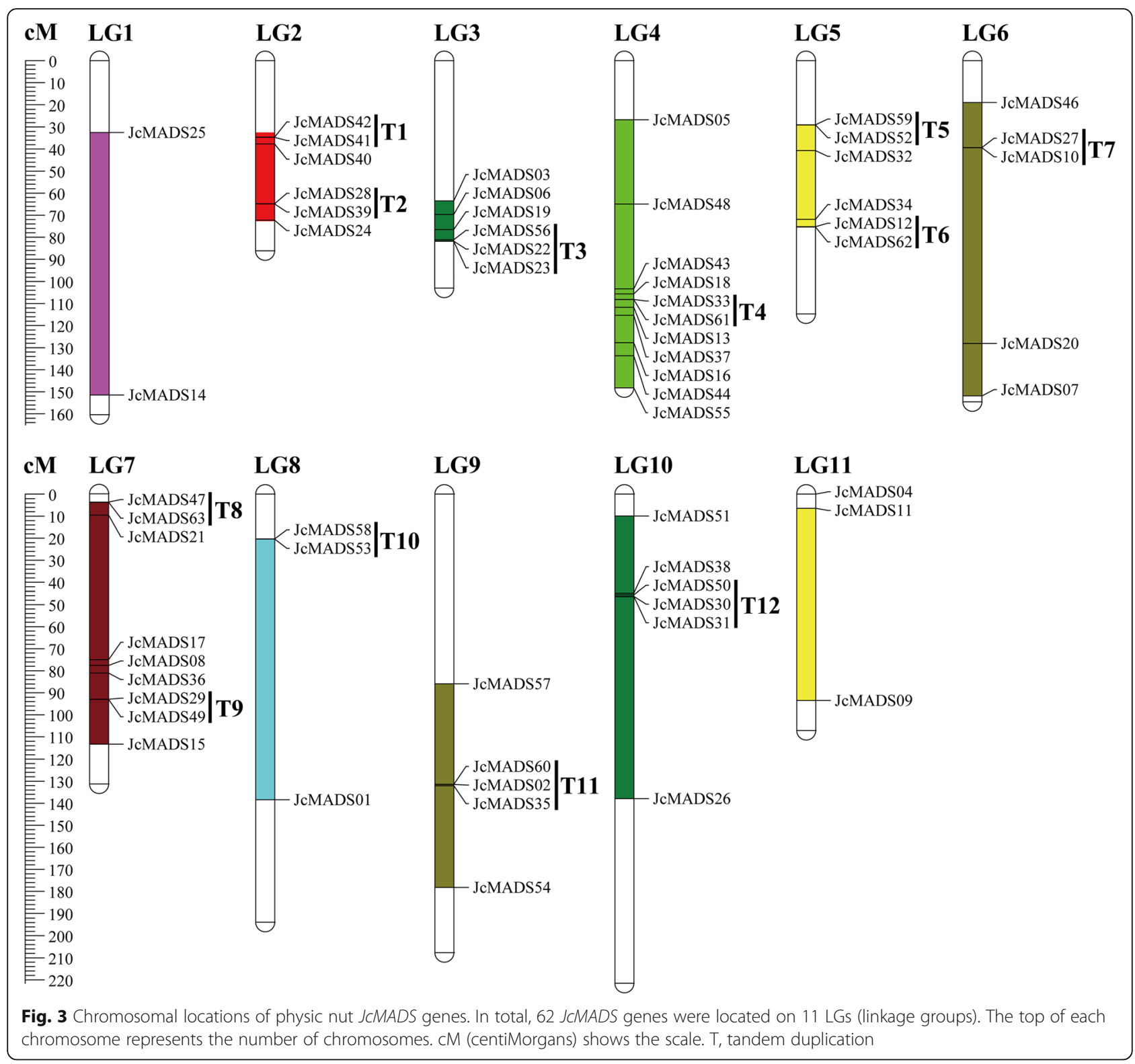

JcMADS genes for which expression was detected, two (JcMADSO3 and 53) were highly expressed across all the tissues sampled, ten (JcMADSO9, 10, 16, 19, 23, 40, 41, 42, 56 and 58) were expressed only in seeds, thirteen (JcMADSO1, $02,05,08,13,29,34,40,44,51,55,60$, and 63) exhibited highest expression in seeds, four (JcMADS24, 36, 48 and 49) preferred to be expressed in roots, and one (JCMADS32) was most strongly expressed in the stem cortex.

Most JcMADS genes had higher expression in seeds at the S1 stage than at the S2 stage (Fig. 4). It was noteworthy that nine genes (JCMADS01, 09, 10, 23, 40, 42, $55,56,48$ and 58) was examined expression only in seeds at S1 stage (Fig. 4). Based on the results of expression pattern analysis, the JcMADS4O gene was chosen for functional research.

\section{Expression profile of JCMADS under abiotic stress} conditions

Many studies have suggested that some MADS-box genes encode proteins participated in the regulation of abiotic stresses [21, 28, 29]. We therefore further investigated the patterns of expression of JCMADS genes in leaves after $2 \mathrm{~d}, 4 \mathrm{~d}$ and $7 \mathrm{~d}$ of drought stress and after $2 \mathrm{~h}, 2 \mathrm{~d}$ and $4 \mathrm{~d}$ of salinity stress according to data from RNA-seq. Our data suggested that the transcript abundances of seven JcMADS genes indicated at least a twofold enhancement or reduction compared with the control in response to at least one stress at one or more time points (Fig. 5). Of these seven genes detected as having differential expression, three (JcMADS29, 36 and 53) exhibited significantly induced or inhibited 


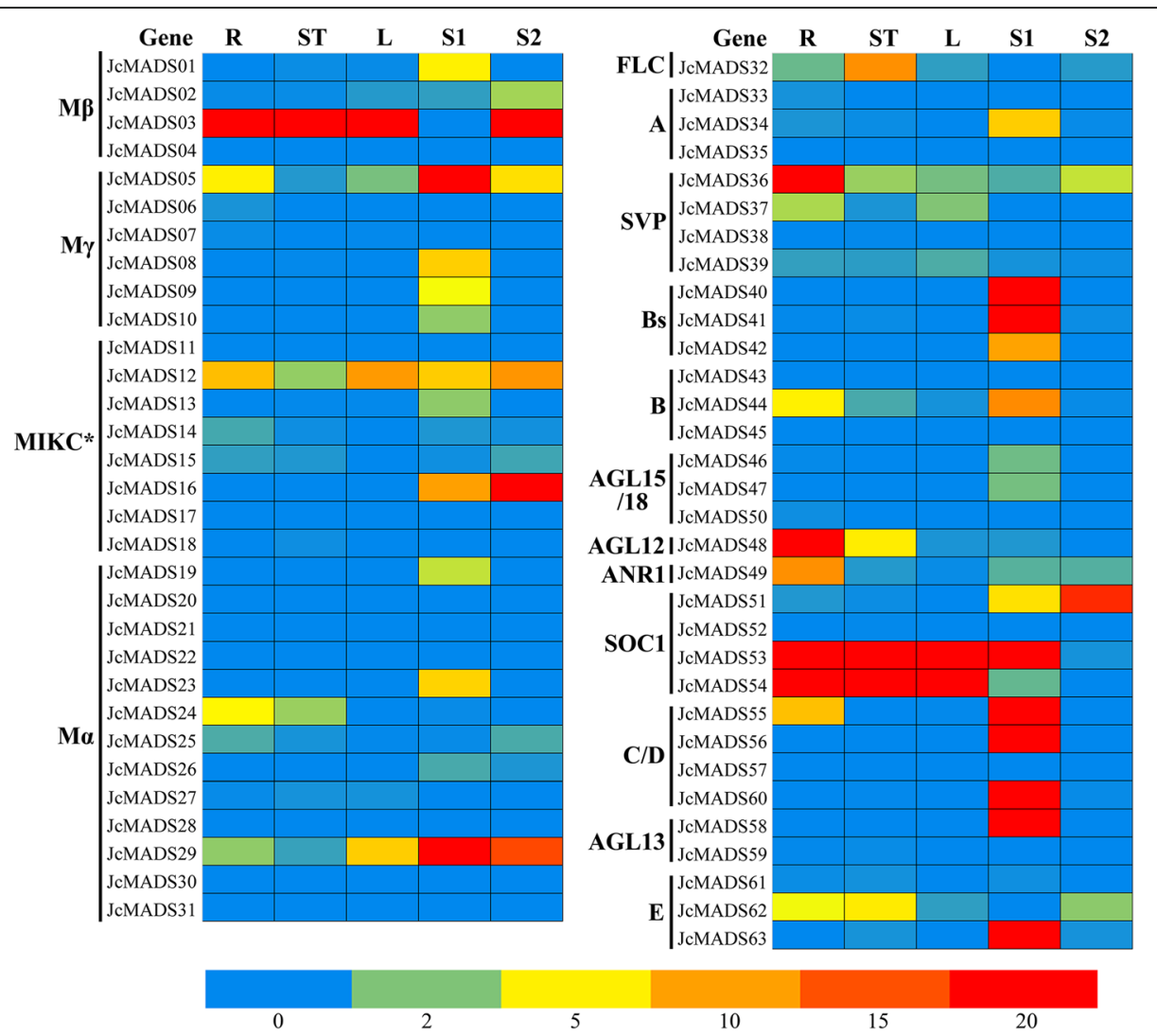

Fig. 4 Patterns of expression of each JCMADS gene in physic nut roots (R), stem cortex (ST), leaves (L), S1(seeds at an early developmental stage) and $\mathbf{S 2}$ (seeds at filling stage), the levels of expression are displayed at the bottom by a colored scale

expression in response to drought and salinity stresses, three (JcMADS12, 37 and 54) showed differential expression only in response to drought stress, and JcMADSO5 was solely affected by salt stress.

\section{JcMADS40 gene encodes a transcriptional activator located in the nucleus}

To confirm the subcellular localization of the protein encoded by JcMADS40 gene, the 35S:JcMADS40-YFP fusion construct and the 35S:YFP empty vector were introduced into Arabidopsis protoplasts. The fluorescence signals from the protoplasts were then observed immediately by confocal laser-scanning microscopy. As shown in Fig. 6, we observed that the yellow fluorescent signal was distributed throughout the whole of the cell when the 35S: YFP vector was used, whereas in protoplasts harboring the construct 35S:JcMADS40-YFP a strong yellow fluorescent signal was detected in the nuclei. These findings indicate that JCMADS40 gene is located in the nucleus.

A dual-luciferase assay was used to examine the transcription activation activity of JcMADS40 protein. The full-length CDS of JcMADS40 was attached to the pBD vector, then the $\mathrm{pBD}$-JcMADS40 fusion effector vector and the $\mathrm{p} 5 \times \mathrm{GAL}-$ Reporter vector were transformed into Arabidopsis protoplasts. The results indicated that the LUC/REN ratio was obviously lower in the control protoplasts $(\mathrm{pBD})$ than in the pBD-JcMADS40 group. Our data suggest that the full-length JcMADS40 has transactivation activity (Fig. 7). Based on the above results, we drew the conclusion that JcMADS40 functions as a transcription activator.

\section{Phenotypic analysis of transgenic rice plants expressing JCMADS40}

To investigate the role of $J C M A D S 40$ in regulating plant development, and to assess the feasibility of using JcMADS genes to control seed size in model crop, we further tested the effects of this gene in rice. Three independent transgenic lines (OE1, OE2 and OE3) were confirmed as expressing JCMADS40 expression using semi-quantitative RT-PCR, and selected for further study. Expression of JcMADS40 were detected in transgenic lines, whereas no expression was found in WT (wild-type) plants (Fig. 8c). Our results showed that the development and flower structure of transgenic plants overexpressing JCMADS40 were very similar to those of WT lines (Fig. 8a and b). Statistical analysis indicated that there was no obvious difference in root and shoot lengths in the JcMADS40 transgenic lines compared to the WT lines 

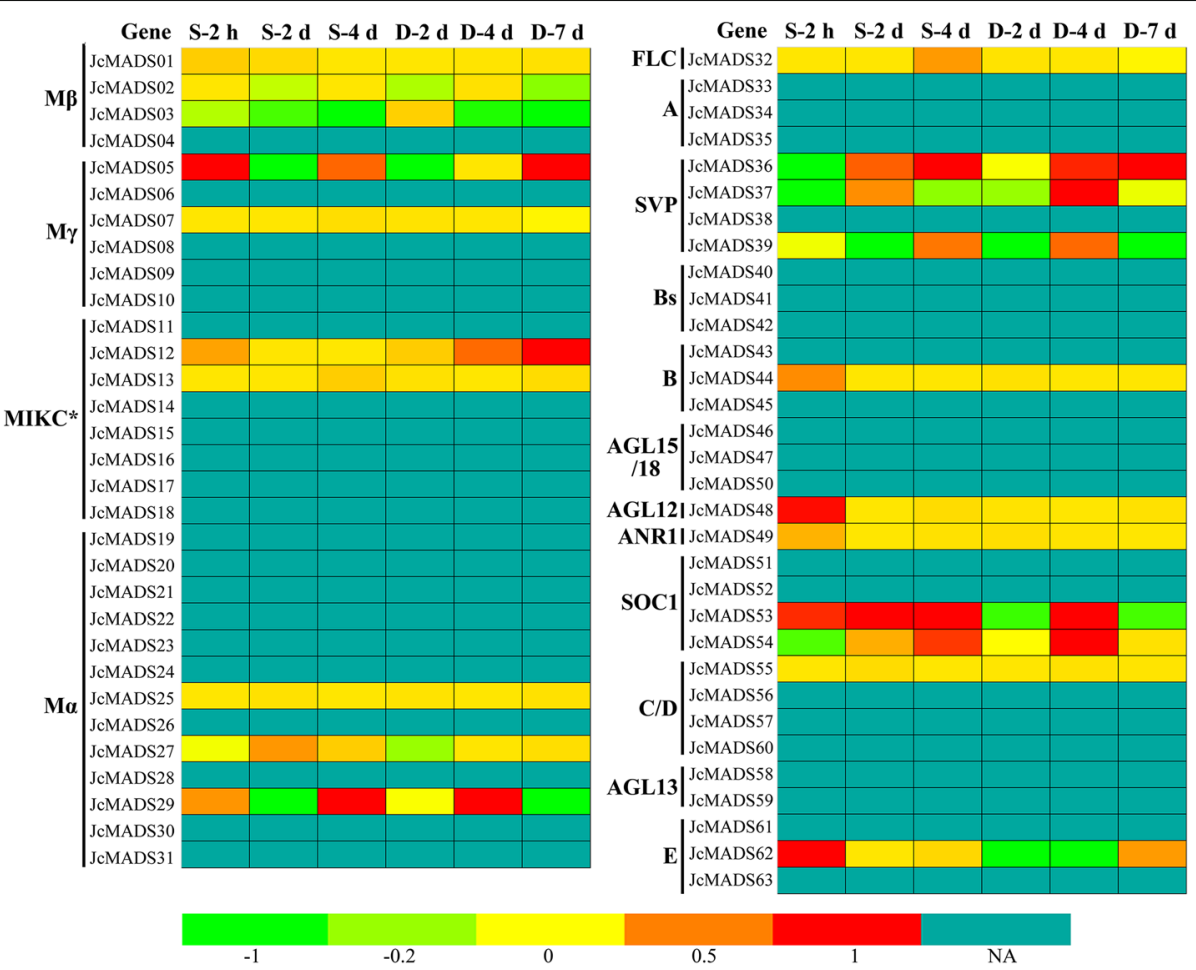

Fig. 5 Levels of expression of the 63 JCMADS genes in physic nut leaves exposed to drought and salinity stresses: $\log _{2}$ ratios of signals from treated versus control leaves are presented as a heat map according to RNA-seq data, the color scale displayed at the bottom is used to display this value. Not available is indicated by NA

(Fig. 8d and e). Taken together, these results led to the conclusion that JCMADS40 did not have any major effect on the growth of the JCMADS40 transgenic plants.

\section{Overexpression of JCMADS40 reduces the grain size in transgenic rice}

As described above, JcMADS40 expression was most strongly detected in seed, suggesting that JcMADS40 might have significant roles in seed development. To verify this, we tested the effects of increasing JcMADS40 expression on rice grain size. We found that JcMADS40 transgenic plants produced dramatically smaller seeds than the WT lines (Fig. 9a). Our data also showed that JcMADS40 overexpression plants had a significant reduction in both grain length and width compared to the WT plants (Fig. 9b and c). We also detected a significant

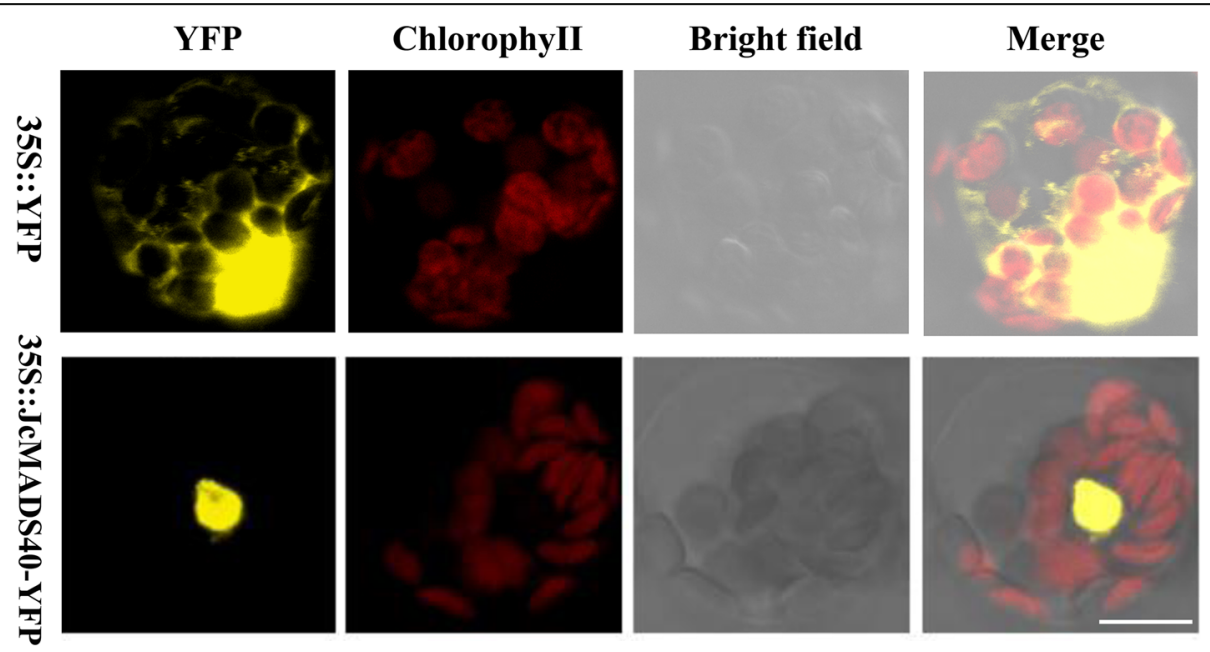

Fig. 6 The product of the JCMADS40 gene is localized in the nucleus. Scale bar, $10 \mu \mathrm{M}$ 

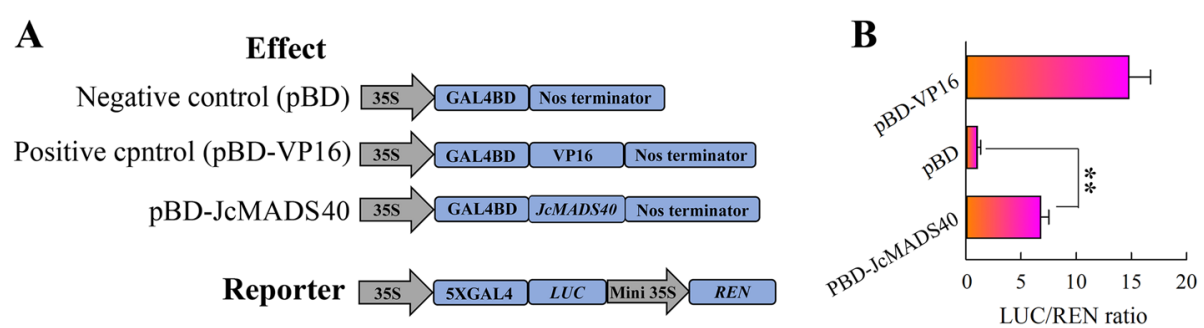

Fig. 7 Transcriptional activity analysis of JCMADS40 in Arabidopsis protoplasts. a Construction of reporter and effector vectors. b A dual-luciferase experiment indicated that JCMADS40 functions as a transcription activator. Each assays included three biological replicates, and each contained two technical replicates (means of $n=6 \pm$ SD; significant differences from controls $(p<0.01)$ are displayed with double asterisks above the bars)

reduction in 1000-seed weight, and yield per plant in JcMADS40 transgenic lines (Fig. 9d and e). Our data suggested that overexpressing JcMADS40 significantly altered seed size in transgenic plants.

To study the molecular mechanism of JcMADS40 gene regulates grain size, we further tested the expression of grain-size-related genes (Fig. 9f). The results showed that expression of some positive regulatory factors, such as GS2, SMG11, was significantly lower in transgenic plants than that in wild-type, while expression of some negative regulatory factors, such as OsMKP1, GW2, was obviously higher than that in wild-type. Taken together, these data supported a putative role for JcMADS genes in seed development.

\section{Discussion}

Increasing evidence suggests that members of the MADS-box family participate in a series of plant physiological phenomena. Up to now, many studies on the role of the MADS-box proteins are mainly concentrated in the model plants rice and Arabidopsis [27]. The molecular mechanisms of seed development in the biofuel plant physic nut, and more specifically the identities, expression patterns and functions of physic nut's MADS-box proteins remain poorly understood. We therefore characterized and tested the expression patterns of MADSbox genes in physic nut, and chose one, JcMADS40, which was most strongly expressed in seed for further functional analysis by overexpressing this gene in rice.

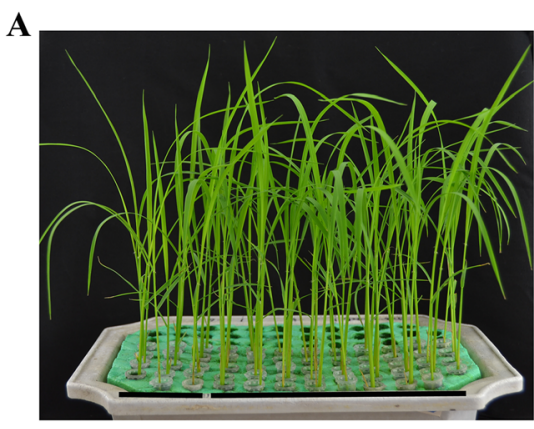

WT OE1 OE2 OE3

D

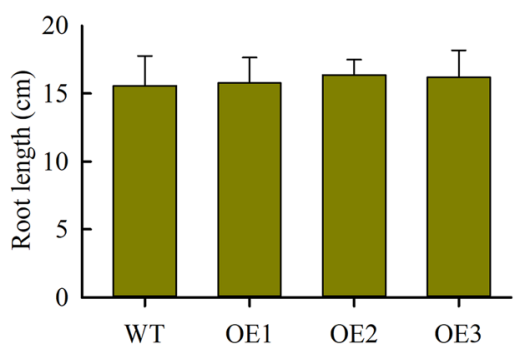

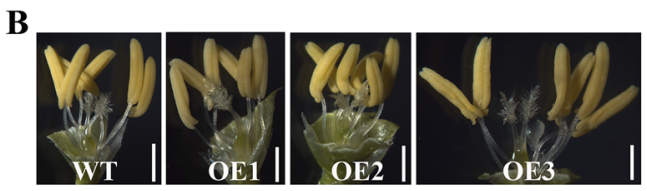
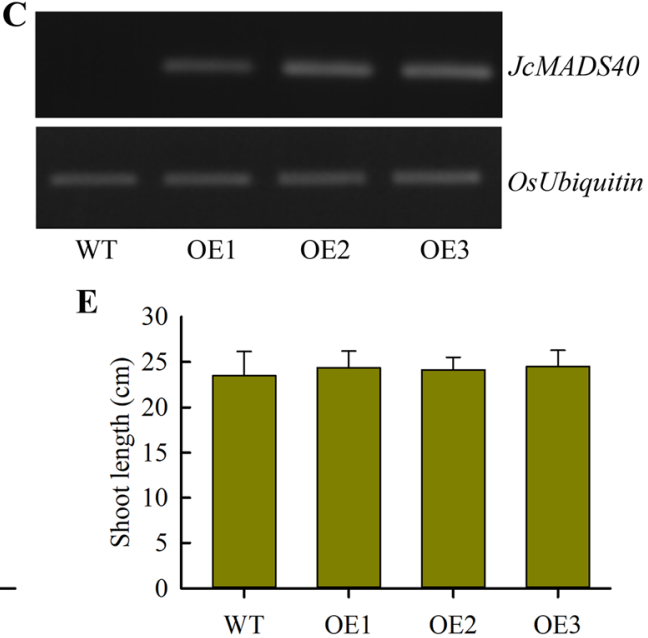

Fig. 8 Characterization of JCMADS40 overexpressed crops (OE1, OE2 and OE3) and their phenotypes. a Growth phenotype of two-week-old wildtype (WT) and JCMADS40 overexpressed lines under exposure to non-stress conditions. b Flower structure in WT and overexpressed crops. $\mathbf{c}$ Levels of JCMADS40 transcript in WT and overexpressed crops. $\mathbf{d}$ Root length in two-week-old WT and overexpressed crops. e Shoot length in two-week-old WT and overexpressed crops. Data presented in (d) and (e) are the means of $n=30 \pm$ SD from three biological assays 

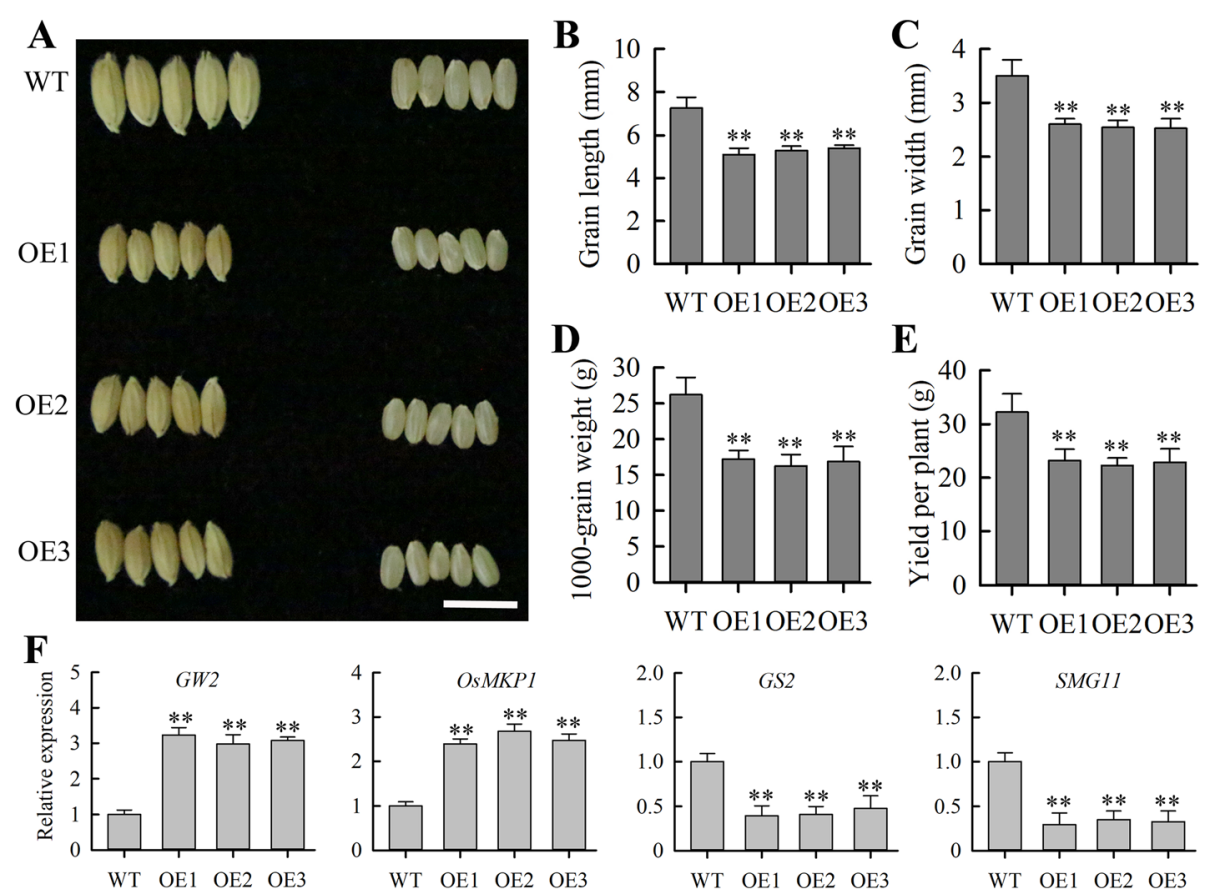

Fig. 9 JCMADS40 regulates grain size. a Grains from wild-type (WT) and JCMADS40 overexpressed crops. Scale bar, $1 \mathrm{~cm}$. $\mathbf{b}$ Grain length in WT and JCMADS40 overexpressed crops. c Grain width in WT and JCMADS40 overexpressed crops. d 1000-grain weight. e Yield per plant. All statistical results in $\mathbf{b}$-e were calculated on plants grown at a spacing of $16 \times 20$ spacing in paddies under non-stress conditions. Data presented in (b), (c), (d) and (e) are the means of $\mathrm{n}=30 \pm \mathrm{SD}$ (the double asterisks indicate $P<0.01$ from the Duncan test). $\mathbf{f}$ Levels of seed-size-lated genes transcript. Each assay contained three biological replicates, and each contained two technical replicates (means of $\mathrm{n}=6 \pm$ SD; significant differences from controls $(p<0.01)$ are displayed with double asterisks above the bars)

In our research, a total of 63 MADS-box genes were confirmed in physic nut. It seems that the physic nut MADS-box family has a smaller number of members (genome size $320 \mathrm{Mb}$ ) compared to those in rice (genome size $466 \mathrm{Mb}$ ) and Arabidopsis (genome size 125 $\mathrm{Mb})$ [25]. A possible reason for this fewer JcMADS genes is likely to be that MADS-box family members in the rice and Arabidopsis genome experience a chromosomal segment duplication event during the early evolution of these species $[9,10]$, whereas members of the physic nut MADS-box family do not experience such duplications [25]. Our phylogenetic tree showed that there were twenty-two MADS genes in the $\mathrm{M} \beta$ group in Arabidopsis, whereas there were only four JcMADS genes in group $M \beta$ (Fig. 1). These finding suggests that the members of this group may have been either acquired in the Arabidopsis lineage or lost in the physic nut after divergence from the last common ancestor shared by Arabidopsis and physic nut. Motif analysis indicated that the distribution of motifs of the protein encoded by the $M A D S$ genes was diverse among different groups, whereas these members in the same group had a similar motif complement (Fig. 2), supporting their impregnable evolutionary conservation. Similar results have been observed in a variety of plants, including bread wheat [30], potato [12], moso bamboo [13], Arabidopsis [9] and sheepgrass [14]. Our results further show that the evolution and classification of the members of MADS-box family in physic nut are very conserved, as it is in other crops.

Preliminary predictions about the biological functions of genes and their products can be made by analyzing their expression profiles, we therefore detected the expression of 63 MADS-box genes sequencing-based transcriptome data. Our results show that JcMADS55 expression was highest in seeds. Its Arabidopsis homolog AGL11(STK) is essential for seed development [15], and its homolog in oil palm SHELL controls oil production from seeds [31]. It can therefore be inferred from the high transcriptional abundance of JcMADS55 in physic nut seeds that it may participated in regulation of the development of physic nut seed. JcMADS48 had the highest transcription abundance in roots, and in Arabidopsis, its homologous AGL12 is also preferentially expressed in root tissues and is essential for root development $[32,33]$. The results indicated that JcMADS48 may play an important role in physic nut root development. TT16, a MADS-box transcription factor, which affects seed development [34], and its homolog JcMADS40 preferred to express in seeds, suggesting that JcMADS40 
may have a significant regulatory role in seed development. SEPALLATA (SEP) genes have been shown to have a key regulatory role in fleshy fruits development. Data related to fruit development and maturity are available in many plants, such as strawberry [35], bilberry [36], tomato [37], and others. The SEP homolog $J c M A D S 63$, was preferentially expressed in seeds, exhibiting that JcMADS63 may play an important role in physic nut fruit development. JcMADSO3 and 53 were expressed in all tested physic nut tissues (Fig. 4), indicating that the functions of these genes may be throughout the development of physic nut plant. It is worth noting that many JCMADS genes showed preferential expression in seeds, implying that they may all be very important for physic nut plants in seed development. Overall, we deduce that $J_{C M A D S}$ genes may have functions in each growing stages of physic nut plants; further research is required to confirm their roles.

Research increasingly have suggested that MADS-box genes participate in responses to various abiotic stresses in many crop species [21, 28, 29]. For example, CaMADS, which is strongly induced by salinity stress, and by ABA, acts as a mediator that has positive feedback effects in the process of pepper coping with cold, and salinity stress [21]. OsMADS26 is a negative regulator in rice response to drought stress [29]. AGL22 gene plays a crucial role in connecting changes in the initiation of drought stress and primary metabolism [38]. Although some researches have begun to screen certain MADS-box genes as important molecular components of drought and salinity stress responses, we have hitherto lacked complete information about the responses of members of MADS-box family to abiotic stresses (drought and salinity, etc.) in physic nut. In this study, RNA-based sequencing data in response to drought and salt stress, combined with qRT-PCR analysis, enabled us to screen JcMADS genes that respond to abiotic stress. For example, expression of JcMADS29, 36 and 53 was induced or inhibited by salt and drought stresses at one or more time points, while JcMADS12, 37 and 54 responded only to drought stress (Fig. 5). Collectively, we preliminary judgment these $J c M A D S$ genes may have significant roles in the regulation of abiotic stress responses in physic nut, and their functions merit further investigation.

Grain size is composed of grain width, grain length and grain thickness, which is an important agronomic trait that determines crop yield [39]. Although some genes involved in the regulation of seed size have been functionally studied in crop plants [40], the molecular mechanisms regulating rice seed size are still poorly understood. Additionally, TT16 and GOA, two genes of the Arabidopsis B-sister group, have been cloned and functionally analyzed [33, 41, 42]. Previous studies have shown that TT16 gene plays an important regulatory role in inner integument differentiation [41], and GOA plays a role as a negative regulatory factor in seed development [34, 42]. For example, increasing GOA expression significantly reduces seed size, on the contrary, decreasing the gene expression enhances seed size compared to wild-type [42]. In rape, BnTT16 gene has been shown to be expressed mainly in seeds of early development, and the gene is necessary for seed development [43]. These findings together show that B-sister genes have an important regulatory role in seed development. In our work, we observed that a B-sister gene from the MIKC ${ }^{\mathrm{C}}$ group, JcMADS40, had the highest expression in physic nut seeds (Fig. 4), and in order to verify its potential function, we further explored its roles in model crop rice. Rice as a monocot is more distantly related than physic nut and Arabidopsis, both Eudicots. Since B-sister function in rice and Arabidopsis is conserved [44], I agreed, it was valid to use rice. Therefore, in addition to demonstrating the function of the JcMADS genes, we also took this opportunity to verify the feasibility of using these genes to regulate seed development in rice crops. Our results showed that the JcMADS40 transgenic plants had smaller, shorter and narrower grains and lower 1000-grain weight compared with wild-type plants (Fig. 9). Furthermore, our results also suggested that JcMADS40 overexpressing plants reduced expression of GS2 and SMG11, and increased expression of OsMKP1 and GW2 (Fig. 9f). SMG11 overexpressing crops enhances grain size by up-regulating or down-regulating the transcriptional abundance of some grain-size-related genes [39]. Plants that increase OsMKP1 expression have smaller grain compared to wild-type, conversely, results in larger grain [45]. GW2 gene mutation enhances grain size and grain weight [46]. GS2 acts as a positive regulator in the regulation of grain size and weight [46]. In short, JcMADS40 overexpressing crops have smaller grains at least in part by affecting the transcription of these genes. These findings further support a role for JcMADS40 in negative regulation of grain size. Reporter gene assays indicated that JcMADS40 may function as a transcriptional activator, its role in negatively mediating grain size is likely to be regulated by the activation of other suppressors. In summary, the results can provide new gene resources for future explore the regulatory role of MADS-box family members in physic nut, especially with respect to their effects on seed size.

\section{Conclusions}

In our research, we identified $63 J c M A D S$ genes in physic nut, and characterized their expression profiles under normal growth and abiotic stress conditions. Transgenic plants that overexpress one of the members of the MADS-box family (JcMADS40) have a smaller 
grain, lower 1000-grain weight and yield per plant compared to wild-type plants, supporting this speculation that some JcMADS genes are involved in the regulation of seed development in physic nut. These findings can provide some valuable references in order to predict the function of MADS-box genes in stress tolerance and seed development, and comprehensive analysis of the gene family produced results that will be helpful in screening genes for further functional research and for the molecular improvement of yield traits in physic nut.

\section{Methods}

\section{Plant materials}

An inbred cultivar of $J$. curcas, GZQX0401, was used in this study owing to its genome sequencing has been completed [26]. The physic nut seeds in our research came from South China Botanical Garden, Chinese Academy of Sciences, Guangzhou, China. ZH11 (japonica cv. Zhonghua 11) was used as wild-type rice (Oryza sativa L.). Seed germination and cultivation of rice crops were carried out in pots filled with soil in the greenhouse of Zhoukou Normal University (China) under natural sunlight.

\section{Identification of MDAS-box gene in physic nut}

One hundred and eight previously identified Arabidopsis MADS-domain protein sequences were used as queries in a search against the physic nut genome database. In addition, the HMM profile of the conserved MADSdomain (PF00319), obtained from the website of Pfam (Pfam 32.0, http://pfam.xfam.org/), was employed to carry out a BLASTP search against the physic nut database using the e-value (expected value) cut-off set at 0.01 . All possible proteins identified as containing the MADS domain were confirmed through the SMART service (http://smart.embl-heidelberg.de/). The theoretical pI and molecular weight of all confirmed JcMADS proteins were determined using the ExPASy ProtParam tool (http://expasy.org/).

\section{Phylogenetic analysis}

MADS protein sequences from Arabidopsis were downloaded from the TAIR database (https://www.arabidopsis.org/), and sequences for rice and Jatropha curcas were downloaded from GenBank (http://www.ncbi.nlm. nih.gov/). The ClustalX (1.83) program was employed to carry out alignment of multiple amino acid sequences. Phylogenetic trees comparing physic nut, rice and Arabidopsis MADS proteins were constructed using the Maximum likelihood method according to the similarity of full-length amino acid sequence, and the results were obtained with the IQ-TREE [47].

\section{Conserved motif and chromosomal distribution}

The conserved motifs of individual MADS-domain proteins were identified using the MEME server (http://alternate.meme-suite.org/). MEME was performed according to the following requirements: site distribution (Zero or one occurrence per sequence), motif number (20), motif width (between 6 and 100 wide). Chromosomal locations of JcMADS proteins were obtained as described by Wu [26], and MapChart software package was employed to draw the linkage maps of JcMADS proteins.

\section{Expression profile analysis of JCMADS genes}

Roots, stems and leaves of three-week-old physic nut seedlings, and seeds of 14 and 35 days after pollination from the same plants, were preserved and employed for further qRT-PCR and RNA-seq analysis. Three-week-old physic nut seedlings (six-leaf stage) were used for salt and drought stress treatments. The specific operations were as follows, the seedlings were directly irrigated with Hoagland solution containing $100 \mathrm{mM} \mathrm{NaCl}$ for salt stress, whereas watering was stopped for drought stress. And then leaves $2 \mathrm{~d}, 4 \mathrm{~d}$, and $7 \mathrm{~d}$ after drought stress, and $2 \mathrm{~h}, 2 \mathrm{~d}$, and $4 \mathrm{~d}$ after salt stress were preserved for subsequent analysis. Raw sequence data were obtained according to standard protocols, and uploaded to the SRA (sequence read archive) at NCBI (accession nos. Are PRJNA257901 (for the drought stress data) and PRJNA244896 (for the salt stress data)). The Illumina gene expression sample preparation kit was employed to prepare tag libraries of all RNA samples, and the Shenzhen BGI's Illumina GAII platform was used for sequencing analysis. Next, the number of expressed tags was counted, then it was normalized to number of transcripts per million tags (TPM), and finally the level of gene transcript was determined. IDEG6 (http://telethon.bio.unipd. it/bioinfo/IDEG6_form/index.html) was employed to identify differentially expressed genes based on previous tests, applying the following requirements: the significance threshold was 0.01, and Bonferroni Correction [48]. Regarding the calculation method of salt and drought stress, the fold change of gene expression (abiotic stress/control) was twice or more was considered to be up-regulated or down-regulated.

\section{Subcellular localization and transcriptional activation analysis}

The amplified coding region of JCMADS40 without the termination codon was inserted into this vector (pSAT6eYFP-N1) to generate 35S::IcMADS40-YFP. The 35S:: JcMADS40-YFP fusion expression construct and the 35S::YFP empty vector were transferred into Arabidopsis protoplasts using the polyethylene glycol-mediated method. Subcellular localization of the control YFP and 
JcMADS40-YFP fusion proteins was observed under a confocal laser scanning microscope. Arabidopsis protoplasts were obtained following Tang [5].

For the transactivation assay of JcMADS40, the fulllength $J c M A D S 40$ gene was fused to the $\mathrm{pBD}$ vector to generate the construct $\mathrm{pBD}-\mathrm{JcMADS40}$. This construct and the $\mathrm{p} 5 \times$ GAL-Reporter vector were introduced into Arabidopsis protoplasts. A ProteoPrep Total Extraction Sample Kit (Sigma) was employed to isolate total protein of Arabidopsis protoplasts according to the operating instructions of the kit, then the fluorescence activity of proteins was analyzed using the enzyme-labeled instrument. The LUC/REN ratio was used to measure the transcriptional activation of JcMADS40.

\section{Gene cloning and plant transformation}

The coding sequence of JCMADS40 was cloned by RT-PCR technology using the total RNA of physic nut seeds as a template with the JcMADS40-F and JcMADS40-R primers given in Additional File 5, and cloned into the pMD18-T vector. The correct coding sequence of JcMADS40 gene was confirmed by sequencing. And then the coding sequence was excised from the pMD18-T vector after digestion with Kpn I and $X b a \mathrm{I}$, then cloned into the pCAMBIA1301 vector at the Kpn I/Xba I site under the control of the CaMV $35 \mathrm{~S}$ promoter. The constructed plant expression vectors were transferred into Agrobacterium (strain EHA105) by the freezethaw procedure, and strains containing this vectors were transferred into calli of rice cv. ZH11, according to the method of Tang [49].

\section{Phenotypic analysis and evaluation of the yield-related traits}

Thirty individual plants of each of the JCMADS40-overexpressing (OE1, OE2, and OE3) and wild-type lines were employed to measure root and shoot lengths, 1000 -grain weight, grain length and width, and yield per plant and to examine flower structure. Each line contained three independent biological replicates.

\section{RNA isolated and qRT-PCR analysis}

Leaves from 2-week-old wild type and transgenic rice seedlings were collected and stored in ultra-low temperature refrigerator $\left(-80^{\circ} \mathrm{C}\right)$ until required for use. Total RNA from different organs was isolated using a HiPure Plant RNA Mini Kit (Code No.R4151-02, Magen, http://www.magentec.com. cn/about.php). The cDNA synthesized using a PrimeScript ${ }^{\mathrm{m} m}$ IV 1st strand cDNA Synthesis Mix (TAKARA, Beijing, China). qRT-PCR was performed on a Mini Option realtime PCR system (LightCycler 480). Cycling conditions were performed according to the following parameters: $30 \mathrm{~s}$ at $95^{\circ} \mathrm{C}, 5 \mathrm{~s}$ at $95^{\circ} \mathrm{C}, 20 \mathrm{~s}$ at $60^{\circ} \mathrm{C}$, and $20 \mathrm{~s}$ at $72^{\circ} \mathrm{C}$. The reaction was carried out for 40 cycles. We used the $2^{-\Delta \Delta C T}$ method to detect relative transcript abundance, and rice
OsUbiquitin gene was employed for normalization. The primers used employed in Additional File 5.

\section{Statistical analysis}

In the research, each experiment contained three biological replicates. Statistical analysis was performed using SAS software package according to the Duncan multiple range test [50].

\section{Supplementary information}

Supplementary information accompanies this paper at https://doi.org/10. 1186/s12864-020-6741-7.

Additional file 1 Summary details of 63 JCMADS genes encoding MADSdomain proteins in physic nut.

Additional file $\mathbf{2}$ Summary of JCMADS proteins, and the reported biological functions of their orthologs in Arabidopsis and rice.

Additional file $\mathbf{3}$. The colored box represents the amino acid sequence of each conserved motif from each protein.

Additional file $\mathbf{4}$ Levels of expression of the 63 JCMADS genes in the organs detected (root, stem cortex, leaf, and seeds) according to RNA-seq data.

Additional file 5. Primers used in this study

\section{Abbreviations}

HD: Homeodomain; ORF: Open reading frame; CDS: Coding sequence

\section{Acknowledgements}

We are very grateful to the Key Laboratory of Plant Resources Conservation and Sustainable Utilization of South China Botanical Garden, Chinese Academy of Sciences, for providing seeds from physic nut.

\section{Authors' contributions}

$X B$ and $Y T$ conceived and designed this research. QW, HL, WW, YZ, NB, YG and JD performed these experiments. JW, YX, SL, RH and WC analyzed the data. YT and TY wrote and revised this manuscript. All authors read and agreed to the final manuscript.

\section{Funding}

The Foundation of Henan Science and Technology Commission (Grant No 182102110200) provides support for our research. The foundation can provide funds for JcMDAS protein research, and paper publication.

\section{Availability of data and materials}

Raw data about our research have been uploaded to NCBI's SRA (sequence read archive) (accession nos. Are PRJNA257901 (for the drought stress data) and PRJNA244896 (for the salt stress data)). All JCMADS protein sequences obtained from our research are available at DDBJ/EMBL/GenBank (accession no. AFEW00000000NCBI). Our research related to other relevant data can be found in supplementary information files and published article.

Ethics approval and consent to participate

Not applicable.

\section{Consent for publication}

Not applicable.

\section{Competing interests}

The authors declare that they have no competing interests.

\section{Author details}

${ }^{1}$ Key Laboratory of Plant Genetics and Molecular Breeding, Zhoukou Normal University, Henan, Zhoukou, China. ${ }^{2}$ Henan Key Laboratory of Crop Molecular Breeding and Bioreactor, Henan, Zhoukou, China. ${ }^{3}$ School of Journalism and Communication, Zhoukou Normal University, Henan, Zhoukou, China. 


\section{Received: 30 January 2020 Accepted: 16 April 2020}

\section{Published online: 28 April 2020}

\section{References}

1. Ferrandiz C, Fourquin C. Role of the FUL-SHP network in the evolution of fruit morphology and function. J Exp Bot. 2014;65(16):4505-13..

2. Gong X, Zhao L, Song X, Lin Z, Gu B, Yan J, Zhang S, Tao S, Huang X. Genome-wide analyses and expression patterns under abiotic stress of NAC transcription factors in white pear (Pyrus bretschneideri). BMC Plant Biol. 2019;19(1):161

3. Li SB, Xie ZZ, Hu CG, Zhang JZ. A review of auxin response factors (ARFs) in plants. Front Plant Sci. 2016;7:47.

4. Roy S. Function of MYB domain transcription factors in abiotic stress and epigenetic control of stress response in plant genome. Plant Signal Behav. 2016:11(1):e1117723.

5. Tang Y, Wang J, Bao X, Liang M, Lou H, Zhao J, Sun M, Liang J, Jin L, Li G, et al. Genome-wide identification and expression profile of HD-ZIP genes in physic nut and functional analysis of the JCHDZ16 gene in transgenic rice. BMC Plant Biol. 2019;19(1):298.

6. Xie Z, Nolan TM, Jiang $H$, Yin Y. AP2/ERF transcription factor regulatory networks in hormone and abiotic stress responses in Arabidopsis. Front Plant Sci. 2019;10:228.

7. Messenguy F, Dubois E. Role of MADS box proteins and their cofactors in combinatorial control of gene expression and cell development. Gene. 2003;316:1-21.

8. Smaczniak C, Immink RG, Angenent GC, Kaufmann K. Developmental and evolutionary diversity of plant MADS-domain factors: insights from recent studies. Development. 2012;139(17):3081-98.

9. Verelst W, Saedler H, Munster T. MIKC* MADS-protein complexes bind motifs enriched in the proximal region of late pollen-specific Arabidopsis promoters. Plant Physiol. 2007;143(1):447-60.

10. Arora R, Agarwal P, Ray S, Singh AK, Singh VP, Tyagi AK, Kapoor S. MADSbox gene family in rice: genome-wide identification, organization and expression profiling during reproductive development and stress. BMC Genomics. 2007:8:242

11. Schilling S, Kennedy A, Pan S, Jermiin LS, Melzer R. Genome-wide analysis of MIKC-type MADS-box genes in wheat: pervasive duplications, functional conservation and putative neofunctionalization. New Phytol. 2019;225:51129.

12. Gao H, Wang Z, Li S, Hou M, Zhou Y, Zhao Y, Li G, Zhao H, Ma H. Genomewide survey of potato MADS-box genes reveals that STMADS1 and StMADS13 are putative downstream targets of tuberigen StSP6A. BMC Genomics. 2018;19(1):726.

13. Zhang $Y$, Tang D, Lin X, Ding M, Tong Z. Genome-wide identification of MADS-box family genes in moso bamboo (Phyllostachys edulis) and a functional analysis of PeMADS5 in flowering. BMC Plant Biol. 2018;18(1):176.

14. Jia J, Zhao P, Cheng L, Yuan G, Yang W, Liu S, Chen S, Qi D, Liu G, Li X. MADS-box family genes in sheepgrass and their involvement in abiotic stress responses. BMC Plant Biol. 2018;18(1):42.

15. Chiara M, Marta Adelina M, Elisabetta C, Arp S, Kater MM, Raffaella B, Lucia C. The MADS box genes SEEDSTICK and ARABIDOPSISB sister play a maternal role in fertilization and seed development. Plant J. 2012;70(3):409-20.

16. Chen C, Begcy K, Liu K, Folsom JJ, Wang Z, Zhang C, Walia H. Heat stress yields a unique MADS box transcription factor in determining seed size and thermal sensitivity. Plant Physiol. 2016;171(1):606-22.

17. Kang I-H, Steffen JG, Portereiko MF, Lloyd A, Drews GN. The AGL62 MADS domain protein regulates cellularization during endosperm development in Arabidopsis. Plant Cell. 2008;20(3):635-47.

18. Lee JH, Ryu HS, Chung KS, Pose D, Kim S, Schmid M, Ahn JH. Regulation of temperature-responsive flowering by MADS-box transcription factor repressors. Science. 2013;342(6158):628-32.

19. Balanza V, Martinez-Fernandez I, Ferrandiz C. Sequential action of FRUITFULL as a modulator of the activity of the floral regulators SVP and SOC1. J Exp Bot. 2014;65(4):1193-203.

20. Pelaz S, Ditta GS, Baumann E, Wisman E, Yanofsky MF. B and C floral organ identity functions require SEPALLATA MADS-box genes. Nature. 2000; 405(6783):200-3.

21. Chen R, Ma J, Luo D, Hou X, Ma F, Zhang Y, Meng Y, Zhang H, Guo W. CaMADS, a MADS-box transcription factor from pepper, plays an important role in the response to cold, salt, and osmotic stress. Plant Sci. 2019;280: 164-74.
22. Wang Z, Wang F, Hong Y, Yao J, Ren Z, Shi H, Zhu JK. The flowering repressor SVP confers drought resistance in Arabidopsis by regulating abscisic acid catabolism. Mol Plant. 2018:1184-97.

23. Guo X, Chen G, Cui B, Gao Q, Hu Z. Solanum lycopersicum agamous-like MADS-box protein AGL15-like gene, SIMBP11, confers salt stress tolerance. Mol Breeding. 2016;36(9):125.

24. Openshaw K. A review of Jatropha curcas: an oil plant of unfulfilled promise. Biomass Bioenergy. 2000;19(1):1-15.

25. Li C, Wang Y, Xu L, Nie S, Chen Y, Liang D, Sun X, Karanja BK, Luo X, Liu L. Genome-wide characterization of the MADS-box gene family in radish (Raphanus sativus L.) and assessment of its roles in flowering and floral organogenesis. Front Plant Sci. 2016;7:1390.

26. Wu P, Zhou C, Cheng S, Wu Z, Lu W, Han J, Chen Y, Chen Y, Ni P, Wang Y, et al. Integrated genome sequence and linkage map of physic nut (Jatropha curcas L.), a biodiesel plant. Plant J. 2015;81(5):810-21.

27. Cannon SB, Mitra A, Baumgarten A, Young ND, May G. The roles of segmental and tandem gene duplication in the evolution of large gene families in Arabidopsis thaliana. BMC Plant Biol. 2004;4(1):10.

28. Castelán-Muñoz N, Herrera J, Cajero-Sánchez W, Arrizubieta M, Trejo C, García-Ponce B, Sánchez MP, Álvarez-Buylla ER, Garay-Arroyo A. MADS-Box genes are key components of genetic regulatory networks involved in abiotic stress and plastic developmental responses in plants. Front Plant Sci. 2019;10:853.

29. Khong GN, Pati PK, Richaud F, Parizot B, Bidzinski P, Mai CD, Bes M, Bourrie I, Meynard D, Beeckman T, et al. OsMADS26 negatively regulates resistance to pathogens and drought tolerance in rice. Plant Physiol. 2015;169(4):2935-49.

30. Ma J, Yang Y, Luo W, Yang C, Ding P, Liu Y, Qiao L, Chang Z, Geng H, Wang $P$, et al. Genome-wide identification and analysis of the MADS-box gene family in bread wheat (Triticum aestivum L.). PLoS One. 2017;12(7):e0181443.

31. Singh R, Low ET, Ooi LC, Ong-Abdullah M, Ting NC, Nagappan J, Nookiah R, Amiruddin MD, Rosli R, Manaf MA, et al. The oil palm SHELL gene controls oil yield and encodes a homologue of SEEDSTICK. Nature. 2013;500(7462): 340-4.

32. Burgeff C, Liljegren SJ, Tapia-Lopez R, Yanofsky MF, Alvarez-Buylla ER. MADSbox gene expression in lateral primordia, meristems and differentiated tissues of Arabidopsis thaliana roots. Planta. 2002;214(3):365-72.

33. Pacheco-Escobedo MA, Ivanov VB, Ransom-Rodriguez I, Arriaga-Mejia G, Avila H, Baklanov IA, Pimentel A, Corkidi G, Doerner P, Dubrovsky JG, et al. Longitudinal zonation pattern in Arabidopsis root tip defined by a multiple structural change algorithm. Ann Bot. 2016;118(4):763-76.

34. Prasad K, Ambrose BA. Shaping up the fruit: control of fruit size by an Arabidopsis B-sister MADS-box gene. Plant Signal Behav. 2010;5(7):899-902.

35. Seymour GB, Ryder CD, Cevik V, Hammond JP, Popovich A, King GJ, Vrebalov J, Giovannoni JJ, Manning K. A SEPALLATA gene is involved in the development and ripening of strawberry (Fragaria $x$ ananassa Duch.) fruit, a non-climacteric tissue. J Exp Bot. 2011;62(3):1179-88.

36. Jaakola L, Poole M, Jones MO, Kamarainen-Karppinen T, Koskimaki JJ, Hohtola A, Haggman H, Fraser PD, Manning K, King GJ, et al. A SQUAMOSA MADS box gene involved in the regulation of anthocyanin accumulation in bilberry fruits. Plant Physiol. 2010;153(4):1619-29.

37. Vrebalov J, Pan IL, Arroyo AJ, McQuinn R, Chung M, Poole M, Rose J, Seymour G, Grandillo S, Giovannoni J, et al. Fleshy fruit expansion and ripening are regulated by the tomato SHATTERPROOF gene TAGL1. Plant Cell. 2009;21(10):3041-62.

38. Bechtold U, Penfold CA, Jenkins DJ, Legaie R, Moore JD, Lawson T, Matthews JS, Vialet-Chabrand SR, Baxter L, Subramaniam S, et al. Time-series transcriptomics reveals that AGAMOUS-LIKE22 affects primary metabolism and developmental processes in drought-stressed Arabidopsis. Plant Cell. 2016;28(2):345-66

39. Fang N, Xu R, Huang L, Zhang B, Duan P, Li N, Luo Y, Li Y. SMALL GRAIN 11 controls grain size, grain number and grain yield in rice. Rice (New York, NY). 2016;9(1):64.

40. Li N, Xu R, Li Y. Molecular networks of seed size control in plants. Annu Rev Plant Biol. 2019;70:435-63.

41. Nesi N, Debeaujon I, Jond C, Stewart AJ, Jenkins GI, Caboche M, Lepiniec L. The TRANSPARENT TESTA16 locus encodes the ARABIDOPSIS BSISTER MADS domain protein and is required for proper development and pigmentation of the seed coat. Plant Cell. 2002;14(10):2463-79.

42. Prasad K, Zhang X, Tobon E, Ambrose BA. The Arabidopsis B-sister MADSbox protein, GORDITA, represses fruit growth and contributes to integument development. Plant J. 2010;62(7):203-14. 
43. Chen G, Deng W, Peng F, Truksa M, Singer S, Snyder CL, Mietkiewska E, Weselake RJ. Brassica napus TT16 homologs with different genomic origins and expression levels encode proteins that regulate a broad range of endothelium-associated genes at the transcriptional level. Plant J. 2013; 74(4):663-77.

44. Yang X, Wu F, Lin X, Du X, Chong K, Gramzow L, Schilling S, Becker A, Theissen G, Meng Z. Live and let die - the B (sister) MADS-box gene OsMADS29 controls the degeneration of cells in maternal tissues during seed development of rice (Oryza sativa). PLoS One. 2012;7(12):e51435.

45. Xu R, Yu H, Wang J, Duan P, Zhang B, Li J, Li Y, Xu J, Lyu J, Li N, et al. A mitogen-activated protein kinase phosphatase influences grain size and weight in rice. Plant J. 2018;95(6):937-46.

46. Song XJ, Huang W, Shi M, Zhu MZ, Lin HX. A QTL for rice grain width and weight encodes a previously unknown RING-type E3 ubiquitin ligase. Nat Genet. 2007;39(5):623-30

47. Nguyen LT, Schmidt HA, von Haeseler A, Minh BQ. IQ-TREE: a fast and effective stochastic algorithm for estimating maximum-likelihood phylogenies. Mol Biol Evol. 2015;32(1):268-74.

48. Romualdi C, Bortoluzzi S, D'Alessi F, Danieli GA. IDEG6: a web tool for detection of differentially expressed genes in multiple tag sampling experiments. Physiol Genomics. 2003;12(2):159-62.

49. Tang Y, Bao X, Zhi Y, Wu Q, Guo Y, Yin X, Zeng L, Li J, Zhang J, He W, et al. Overexpression of a MYB family gene, OSMYB6, increases drought and salinity stress tolerance in transgenic rice. Front Plant Sci. 2019:10:168.

50. Duncan DB. Multiple range and multiple F tests. Biometrics. 1955;11(1):1-42.

\section{Publisher's Note}

Springer Nature remains neutral with regard to jurisdictional claims in published maps and institutional affiliations.

Ready to submit your research? Choose BMC and benefit from:

- fast, convenient online submission

- thorough peer review by experienced researchers in your field

- rapid publication on acceptance

- support for research data, including large and complex data types

- gold Open Access which fosters wider collaboration and increased citations

- maximum visibility for your research: over $100 \mathrm{M}$ website views per year

At BMC, research is always in progress.

Learn more biomedcentral.com/submissions 